\title{
Ocular surface cytokine profile in chronic Stevens- Johnson syndrome and its response to mucous membrane grafting for lid margin keratinisation
}

\author{
Srividya Gurumurthy, ${ }^{1}$ Geetha Iyer, ${ }^{2}$ Bhaskar Srinivasan, ${ }^{2}$ Shweta Agarwal, ${ }^{2}$ \\ Narayanasamy Angayarkanni ${ }^{1}$
}

\begin{abstract}
- Additional material is published online only. To view, please visit the journal online (http://dx.doi.org/10.1136/ bjophthalmol-2017-310373).

${ }^{1}$ R.S. Mehta Jain Department of Biochemistry and Cell Biology, KBIRVO Block, Vision Research Foundation, Sankara Nethralaya Chennai, India

${ }^{2}$ C.J. Shah Cornea Services, Dr G Sitalakshmi Memorial Clinic for Ocular Disorders, Medical Research Foundation, Sankara Nethralaya, Chennai, India
\end{abstract}

\section{Correspondence to}

Dr Geetha Iyer, C.J. Shah Cornea Services: Dr G Sitalakshmi Memorial Clinic for Ocular Disorders, Medical Research Foundation, Sankara Nethralaya, Chennai, India; drak@snmail.org and Professor Narayanasamy Angayarkanni, R.S. Mehta Jain Department of Biochemistry and Cell Biology, Vision Research Foundation, Sankara Nethralaya, 41, College Road, Chennai 600006, India; drak@snmail.org

Received 21 February 2017 Revised 4 April 2017 Accepted 1 May 2017 Published Online First 8 July 2017
Check for updates

To cite: Gurumurthy $S$, lyer $\mathrm{G}$, Srinivasan $\mathrm{B}$, et al. Br J Ophthalmol 2018;102:169-176
ABSTRACT
Background To study the tear cytokine and the conjunctival and oral mucosal marker profile in chronic ocular Stevens-Johnson syndrome (SJS) and their alteration following mucous membrane grafting (MMG) for lid margin keratinisation (LMK).

Methods In a 1-year prospective study, SJS cases ( $n=25)$ and age-matched/sex-matched healthy controls $(n=25)$ were recruited. Tear specimen (Schirmer's strip), conjunctival and oral mucosal imprints were collected from controls and SJS cases pre-MMG and post-MMG (at first follow-up, $n=17)$. Tear cytokines were profiled using 27-bioplex array. Transforming growth factor-beta (TGF$\beta)$-mediated extracellular matrix changes in conjunctival and oral mucosal cells were analysed by gene expression studies. 30

Results Tear cytokine profiling of chronic SJS cases at pre-MMG stage revealed significant upregulation of cytokines granulocyte-macrophage colony-stimulating factor (GM-CSF), interleukin (IL)-8, IL-1 $\beta$, monocyte chemoattractant protein-1, IL-15, IL-2, IL-17A and basic fibroblast growth factor (bFGF) with downregulation of IP-10 (interferon gamma-induced protein 10), tumour necrosis factor- $\alpha$, interferon- $\gamma$, IL-10, vascular endothelial growth factor, regulated upon activation normal T-cell expressed and secreted (RANTES), IL-7, IL-12p70 and IL-13, with maximal increase in GM-CSF and maximal downregulation of IP-10, respectively. Of these, IL-2, IL-15, bFGF and IL-17A showed significant correlation with disease severity, pre-MMG. Conjunctival cells preMMG showed increase in TGF- $\beta 1$, TGF- $\beta$ RII, connective tissue growth factor and collagen-III gene expression by $10,67,173$ and 184 folds, respectively, which dropped to $1.3,11,13.5$ and 19 folds correspondingly, postMMG. However, their expressions in oral mucosa were negligible.

Conclusion A proinflammatory, profibrotic, antiapoptotic ocular surface milieu characterises chronic ocular SJS. IP-10, an antifibrotic cytokine was noted to be maximally downregulated, unlike in other forms of chronic dry eye disease. The alterations in the ocular surface are seen to reverse largely with MMG for LMK.

\section{INTRODUCTION}

Stevens-Johnson syndrome (SJS) and toxic epidermal necrolysis (TEN) are relatively rare, acute exfoliative blistering disorders of the skin due to adverse drug reaction causing epidermal necrosis and mucous membrane breakdown, with incidence of ocular complications ranging from $20 \%$ to $77 \%$.
The ocular sequelae of SJS causes visual debilitation due to chronic inflammatory changes occurring on the ocular surface as well as significant functional impairment due to associated symptoms like photophobia, dryness and foreign body sensation. ${ }^{1}$

Lid margin keratinisation (LMK), a feature that occurs in most eyes of patients with SJS has been identified as an important cause for the continued inflammation and deterioration of the ocular surface as published in earlier reports, if not intervened in the acute stage with amniotic membrane grafting. ${ }^{1-3}$ Our earlier study on the comprehensive management for SJS highlighted the beneficial impact of mucous membrane grafting (MMG) for LMK. $^{3}$

A cytokine storm in the tear samples from a patient of acute SJS has been reported earlier. ${ }^{4}$ However, there are no reports on the tear cytokine expression in the chronic phase of SJS. Therefore, this study aims to understand the same in eyes with chronic sequelae of SJS including LMK and their alteration following MMG.

\section{METHODS}

\section{Patients and healthy controls}

A prospective study at a tertiary eye care centre was conducted between May 2014 and April 2015 in adherence to the tenets of the Declaration of Helsinki, with informed consent from study recruits and following approval from the Institutional Review Board. Chronic SJS cases with LMK warranting mucous membrane graft were included in the study. All patients with SJS underwent a detailed ocular examination and the parameters analysed were: best-corrected visual acuity; Schirmer's I test (graded as $1,<5 \mathrm{~mm} ; 2,5-10 \mathrm{~mm} ; 3$, $11-15 \mathrm{~mm}$ and $4,>15 \mathrm{~mm}$ ) and fluorescein corneal staining scores from 0 to 9 . The diagnosis of SJS was based on a confirmed history of acute onset of high fever, serious mucocutaneous illness with skin eruptions and involvement of at least two mucosal sites including the ocular surface. Inclusion criteria included all eyes with LMK that warranted MMG, excluding eyes with total limbal stem cell deficiency, grade 3 or worse symblepharon ${ }^{5}$ and total keratinisation of the ocular surface.

Samples from patients with SJS were collected preoperatively ( $\mathrm{n}=50$ eyes) and postoperatively ( $n=33$ eyes) at their first follow-up visit prior to performing any other ophthalmic investigations which included tear collection, conjunctival imprint 
from four quadrants and oral mucosal imprint cytology from the lip. The enrolled patients were only on artificial tear substitutes preintervention and postintervention at the time of sample collection. Topical antibiotics started postoperatively were stopped at 1 week following surgery. The MMG for LMK was performed as described earlier. ${ }^{3}$

Healthy volunteers ( $\mathrm{n}=50$ eyes), age-matched/sex-matched to the cases, were the control group, selected using inclusion criteria namely, absence of ocular surface-related symptoms; and Schirmer's wetting of $>10 \mathrm{~mm}$ at $5 \mathrm{~min}$. Exclusion criteria included any history of ophthalmic or systemic disease, ocular allergy, ophthalmic surgery and currently under medication/ pregnancy/contact lens wear.

\section{Tear sample collection and extraction}

The tear samples were collected and extracted from the Schirmer's strips according to the method described earlier, except that extraction was done in IX phosphate buffered saline (PBS) with $0.1 \%$ Triton X-100. ${ }^{6}$

\section{Multiplex analysis of cytokines in tear samples}

A Bio-Plex multiplex assay (Bio-Plex Human Cytokine 27-plex panel, Bio-Rad Laboratories, Hercules, California, USA) was used to measure the concentrations of 27 cytokines in tear from patients with SJS and healthy controls. Following extraction, the total protein concentration was adjusted to $500 \mu \mathrm{g} / \mathrm{mL}$ with $1 \mathrm{X}$ PBS and $25 \mu \mathrm{L}$ was taken uniformly for Bio-Plex analysis. The analysis was performed according to the manufacturer's instructions and read in Bio-Plex Reader (Bio-Rad Laboratories). Standard curves were generated using the Bio-Plex Manager System (software V.6.0; Bio-Rad Laboratories) to calculate the cytokine concentrations in the tear samples.

\section{Quantitative PCR}

Total RNA was isolated from the imprints using Tripure RNA isolation reagent (Roche Diagnostics, Basel, Switzerland) according to manufacturer's protocol. One $\mu$ g of DNaseI (New England Biolabs, Ipswich, Massachusetts, USA) treated total RNA from conjunctival or oral mucosal imprints were used for complementary DNA (cDNA) synthesis using iScript cDNA synthesis kit (Bio-Rad Laboratories). The gene-specific primers were designed for the genes 18S rRNA, $\tau \rho \alpha \nu \sigma \phi о \rho \mu \mathrm{v} \gamma \gamma \gamma \rho \circ \omega \tau \eta$ $\phi \alpha \chi \tau \circ \rho-\beta \varepsilon \tau \alpha$ (ТГФ- $\beta)$, TGF- $\beta$ RI, TGF- $\beta$ RII, connective tissue growth factor (CTGF), collagen-III, $\alpha$-smooth muscle actin $(\alpha-$ SMA), vimentin, $B a x, B c l 2, \beta$-actin and $\alpha$-tubulin (see online supplementary table 1 ). Real-time PCR was performed with the Lightcycler 96 (Roche Diagnostics, Switzerland) using the SYBR Green chemistry. The comparative $2^{(-\Delta \mathrm{Ct})}$ method was used to analyse the results of the genes of interest relative to internal control gene (18 rRNA).

\section{Western blot analysis}

Total protein was extracted from the imprints by incubating them in extraction buffer $(10 \mathrm{mM}$ Tris buffer $(\mathrm{pH} 8.0), 0.2 \%$ Triton X-100, 10\% glycerol, 0.5 mM EDTA, 1 mM dithiothreitol with protease inhibitor cocktail) for $10 \mathrm{~min}$ followed by centrifugation at $8000 \mathrm{rpm}$ for $5 \mathrm{~min}$. The total protein concentration was determined using BCA protein assay kit (Pierce Biotechnology, Waltham, Massachusetts, USA) and $30 \mu \mathrm{g}$ was loaded onto 10\% SDS PAGE and transferred onto a polyvinylidene difluoride membrane (Bio-Rad Laboratories). The antibodies used included rabbit anti- $\alpha$-SMA antibody (Abcam, Cambridge, UK), rabbit anti vimentin antibody (Abcam) at a dilution of
1:500 or mouse anti- $\beta$-actin (Santa Cruz Biotechnology (SCBT), Santa Cruz, California, USA) or mouse anti- $\alpha$-tubulin (SCBT) at a dilution of 1:1000. The membrane was washed and incubated with either anti rabbit/anti mouse IgG-HRP conjugated secondary antibody (SCBT). The blots were developed with Clarity TM Western ECL substrate (Bio-Rad Laboratories) and the images captured using FlourChem FC3 gel documentation system (Protein Simple, San Jose, California, USA). Intensity of band was assessed using Image J software (NIH, Bethesda, Maryland, USA). $\alpha$-Tubulin was used as loading control.

\section{Immunofluorescence}

Conjunctival imprints from patients with SJS and controls were fixed with $4 \%$ paraformaldehyde for $20 \mathrm{~min}$ at room temperature. The fixed cells were washed twice with wash buffer $(0.1 \%$ bovine serum albumin (BSA) in $1 \mathrm{X}$ PBS), permeabilised in $1 \mathrm{X}$ PBS $+0.1 \%$ Tween 20 and blocked with $1 \%$ BSA for $30 \mathrm{~min}$ at room temperature. The imprints were then incubated with primary rabbit anti vimentin/ anti- $\alpha$-SMA/ mouse anti- $\alpha$-tubulin (1:200), in dilution buffer (1X PBS, 1\% BSA) at room temperature for 2 hours. The imprints were washed twice and incubated with corresponding fluorescein isothiocyanate-conjugated secondary antibodies (1:500, Invitrogen, Carlsbad, California, USA) for 1 hour in dark. Imprints were washed and counterstained with 4',6-diamidino-2-phenylindole for $3 \mathrm{~min}$ in dark. After phosphate-buffered saline Tween 20 washes, the imprints were mounted on a glass slide using antifade mounting medium (Invitrogen) and observed under confocal microscope.

\section{Collection of conjunctival and oral mucosal imprints}

Imprints from the superior, nasal, inferior and temporal bulbar conjunctiva (pre-MMG/ post-MMG) and lower lip (pre-MMG) were collected according to the method described by Gadkari et al. ${ }^{7} \mathrm{H} \& \mathrm{E}$ staining was done in conjunctival and oral mucosal imprints in a subset of three SJS cases, pre-MMG and at first and second follow-up visit post-MMG to observe the cell phenotype. Similarly, periodic acid Schiff staining was done to assess the presence of goblet cells in the conjunctival imprints.

\section{Statistical analyses}

Data were expressed as mean $\pm \mathrm{SD}$, the median and range. The statistical analyses were performed using SPSS for Windows V.14.0. The Pearson's $\chi^{2}$ test was used to compare the proportions of qualitative variables. D'Agostino-Pearson omnibus K2 normality test was used to check the normal distribution of the data. Student's t-test and the Mann-Whitney U test were used to compare the means of the quantitative variables between two independent groups. The Kruskal-Wallis test was used to compare multiple groups. Fold variation of $>1.2$ folds and in $50 \%$ of cases compared with controls were considered significant. One-way analysis of variance (ANOVA) was used to assess the correlation between causative factor, intervention period or disease duration with SJS severity. A p Value $<0.05$ was accepted as statistically significant.

\section{RESULTS}

Patients with SJS ( $n=25,50$ eyes; 12 males/13 females; mean age: $30.88 \pm 11.42$ years $)$ and healthy controls $(n=25,50$ eyes; 12 males/13 females; mean age: 30.16 \pm 7.77 years) were recruited in the study. The controls were age-matched/sex-matched volunteers (see online supplementary table 1). Of the 25 patients, post-MMG samples were collected in 17 patients (33 eyes) at the first follow-up visit between 2 and 3 months (except 1 at 8 

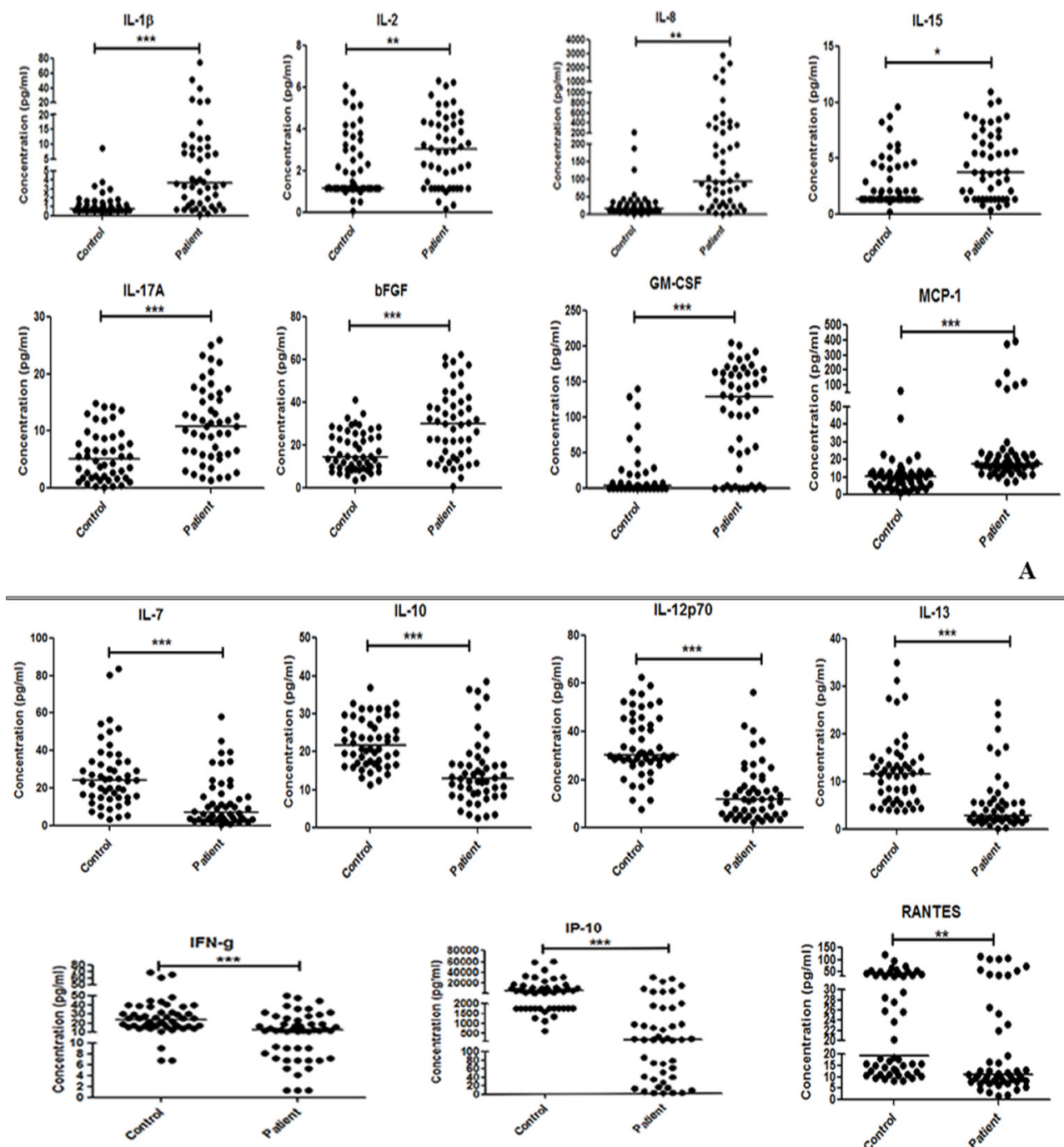

A
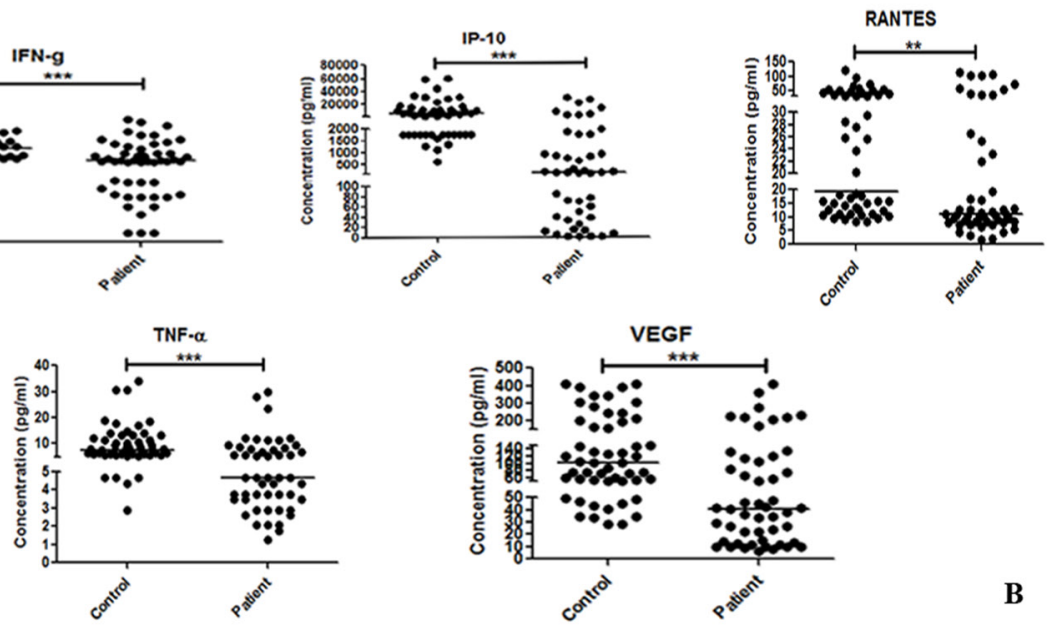

B

Figure 1 Distribution of cytokines in Stevens-Johnson syndrome cases as compared with controls. (A) Overexpressed cytokines. (B) Underexpressed cytokines. ${ }^{* * *} p<0.001$. bFGF, basic fibroblast growth factor; GM-CSF, granulocyte-macrophage colony-stimulating factor; IL, interleukin; IFN- $\gamma$, interferon- $\gamma$, MCP-1, monocyte chemoattractant protein-1; TNF- $\alpha$, tumour necrosis factor- $\alpha$; VEGF, vascular endothelial growth factor.

months) following surgery. After the mucous membrane graft, the corneal fluorescein staining score improved $\geq 3$ in $84.48 \%$ of eyes. The details of demographic/clinical characteristics of the patients are documented in online supplementary table 2 .

\section{Pro-inflammatory and anti-inflammatory cytokine levels} in tears

All the 27 cytokines were detectable in the tear samples (see online supplementary table 3 ). Differential expression analysis of the cytokines in SJS cases prior to MMG revealed eight cytokines to be significantly upregulated and nine to be significantly downregulated (figure 1). The distribution analysis of the upregulated ones revealed maximal increase in GM-CSF and among downregulated ones, IP-10 (interferon gamma-induced protein
10) was maximal. More than $80 \%$ of SJS cases showed upregulated cytokines that were significantly altered (table 1).

On analysing the gender effect on the differential expression, it was observed that IL-6 levels were twofold $(p=0.008)$ increased and RANTES (regulated upon activation normal T-cell expressed and secreted) levels were 2.75 folds $(\mathrm{p}=0.009)$ decreased in the female SJS cases. However, on combining the male and female data, while RANTES was still significantly lower in SJS; IL-6 levels was not significantly increased in patients compared with controls pre -MMG. No other cytokine showed any gender bias.

\section{Cytokine expressions at follow-up after MMG intervention}

An overall improvement of around 48\% (mean 48\% $12 \%$ ) was seen in the levels of all cytokines post-MMG, although IL-7, 


\begin{tabular}{|c|c|c|c|c|}
\hline \multicolumn{5}{|c|}{ SJS cases (preoperative stage) } \\
\hline Cytokine & $\begin{array}{l}\text { Fold change in cytokine Mean } \pm \text { SD } \\
\text { (range) }\end{array}$ & $95 \% \mathrm{Cl}$ & $\begin{array}{l}\% \text { cases above/ below median } \\
\text { of control* }\end{array}$ & $\mathrm{p}$ Value \\
\hline \multicolumn{5}{|c|}{ Increased expression } \\
\hline GM-CSF & $29.02 \pm 19.93(1.02-57.33)$ & 31.43 to 41.41 & 84.22 & $<0.0001$ \\
\hline IL-8 & $17.13 \pm 24.40(1.23-182)$ & 11.27 to 37.29 & 89.51 & $<0.0001$ \\
\hline IL-1 $\beta$ & $13.27 \pm 24.40(1.44-133)$ & 7.17 to 26.28 & 81.58 & $<0.0001$ \\
\hline MCP-1 & $4.77 \pm 8.78(1.02-37.56)$ & 1.99 to 7.12 & 94.77 & $<0.0001$ \\
\hline IL-15 & $2.8 \pm 1.82(1.25-6.08)$ & 2.74 to 3.86 & 76.32 & 0.018 \\
\hline IL-2 & $2.71 \pm 1.46(1.00-4.57)$ & 2.21 to 3.09 & 92.40 & 0.0089 \\
\hline IL-17 & $2.03 \pm 1.20(1.00-4.67)$ & 1.94 to 2.6 & 84.22 & 0.0007 \\
\hline bFGF & $2.0 \pm 1.12(1.08-4.19)$ & 2.04 to 2.66 & 84.22 & $<0.0001$ \\
\hline \multicolumn{5}{|c|}{ Decreased expression } \\
\hline VEGF & $-1.34 \pm 0.92(1.21--14.28)$ & -7.10 to -4.11 & 68.43 & $<0.0001$ \\
\hline TNF- $\alpha$ & $-1.34 \pm 0.52(-1.09--6.08)$ & -2.70 to -1.92 & 73.69 & $<0.0001$ \\
\hline IL-10 & $-1.47 \pm 0.40(-9.09-1.77)$ & -3.14 to -1.92 & 82.97 & $<0.0001$ \\
\hline IFN- $\gamma$ & $-1.52 \pm 0.48(-1.09--20)$ & -5.24 to -2.20 & 76.32 & $<0.0001$ \\
\hline RANTES & $-1.73 \pm 0.56(-3.29-1.79)$ & -3.86 to -2.08 & 73.69 & 0.0010 \\
\hline IL-7 & $-2.02 \pm 0.48(-1.07--50)$ & -6.41 to -2.61 & 81.58 & $<0.0001$ \\
\hline IL-13 & $-2.11 \pm 0.53(-1.06--50)$ & -9.33 to -2.27 & 84.22 & $<0.0001$ \\
\hline IL-12p70 & $-2.12 \pm 0.37(-1.08--16.66)$ & -5.13 to -1.92 & 86.85 & $<0.0001$ \\
\hline IP-10 & $-2.68 \pm 0.94(-1.21--5000)$ & -1012.07 to -168.08 & 86.85 & $<0.0001$ \\
\hline
\end{tabular}

*Above median of control in case of increased expression and below in case of decreased expression level.

bFGF,basic fibroblast growth factor; GM-CSF, granulocyte-macrophage colony-stimulating factor; IL, interleukin;IFN- $\gamma$, interferon- $\gamma$; MCP-1, monocyte chemoattractant protein-1; RANTES, regulated upon activation normal T-cell expressed and secreted; SJS,Stevens-Johnson syndrome; TNF- $\alpha$, tumour necrosis factor- $\alpha$; VEGF,vascular endothelial growth factor.

IL-12p70 and RANTES showed $>20 \%$ improvement (figure 2). IL-15, IL-17A $(\mathrm{p}<0.0001)$ and bFGF $(\mathrm{p}<0.009)$ continued to increase while VEGF continued to decrease $(\mathrm{p}<0.0001)$ significantly post-MMG.

\section{Conjunctival morphology and goblet cell density pre-MMG and post-MMG}

Imprint cytology revealed normal conjunctival epithelial morphology among controls, while those from patients with SJS showed dysplastic cells. The imprints from SJS cases pre-MMG showed negligible goblet cells with improvement in cell numbers post-MMG (see online supplementary figure 1 ).

\section{Evaluation of fibrotic markers in SJS conjunctiva pre-MMG and post-MMG}

Cytokine expression profile in chronic SJS cases pre-MMG was indicative of defective wound healing resulting in fibrosis. The expression of both $\alpha$-SMA and vimentin were higher in the conjunctival imprints of patients with SJS (pre-MMG) indicating myofibroblast formation (figure 3A). The protein expressions of the fibroblast markers, $\alpha$-SMA and vimentin were concomitant to the changes in the corresponding mRNA levels as seen in the conjunctival imprint samples of SJS cases in preintervention and postintervention stages (figure $3 \mathrm{~B}$ ).

As the LMK is addressed by grafting oral mucosal tissue following excision of keratinised lid margin conjunctiva, the gene expression for TGF- $\beta$ signalling was assessed preintervention and postintervention in the conjunctival cells, as well as in the oral mucosa pre-MMG. Accordingly, the expressions of TGF- $\beta 1$ and its receptors, TGF- $\beta$ RI, TGF- $\beta$ RII and the downstream signalling molecules, CTGF and collagen-III were found to be higher in SJS, which reduced post-MMG. This expression pattern was seen in $>56 \%$ of the cases (figure $3 \mathrm{C}(\mathrm{i})$ ). The expressions of TGF- $\beta 1$, CTGF and collagen-III were 4, 16 and 7 folds lower, while that of TGF- $\beta$ RII was 67 times higher in the oral mucosa of SJS cases than in the conjunctiva (figure $3 \mathrm{C}(\mathrm{ii})$ ). Thus, the high TGF- $\beta$ RII expression was differential in the patient's conjunctiva compared with oral mucosa apart from the low TGF- $\beta 1$ and CTGF expression. The expressions of TGF- $\beta 1$, its receptors and downstream molecules studied were negligible in the control mucosa (data not shown).

\section{Evaluation of apoptotic markers in conjunctiva}

As both pro-apoptotic and anti-apoptotic cytokines were low in SJS, mRNA expressions of the apoptotic markers Bax (pro-apoptotic) and Bcl2 (anti-apoptotic) were checked in the conjunctival imprint samples, which showed a net promotion of apoptosis post-MMG (figure 3D).

\section{Clinical correlation}

The correlation between causative factor, intervention period or disease duration with SJS severity was determined using one-way ANOVA. Our results demonstrated that the SJS severity significantly correlated with the disease duration, but not with the causative factor or intervention period. Spearman's rankorder correlation coefficients were used to assess the relationship between the concentrations of the assayed cytokines and SJS severity, which revealed that IL-2, IL-15, IL-17A and bFGF significantly correlate with the disease severity, at the preintervention stage $(\mathrm{p}<0.05)$.

\section{DISCUSSION}

There are no earlier published reports on the tear cytokine profile in chronic SJS. Cutaneous lesions have been examined and reported. ${ }^{8}$ Previous report of selected tear cytokines 
Clinical science
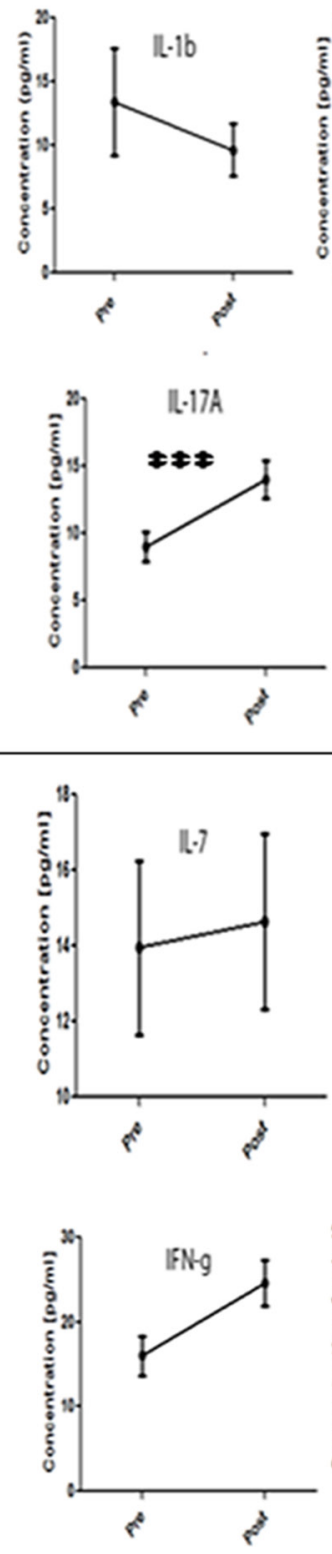
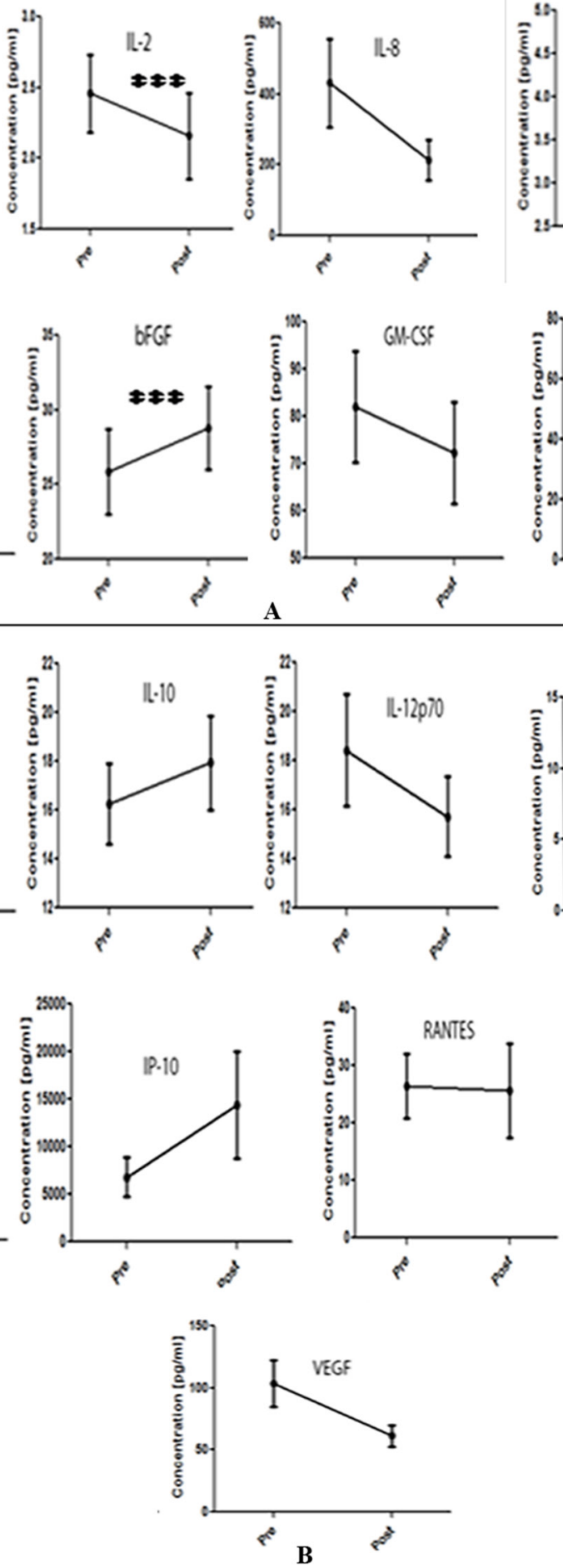
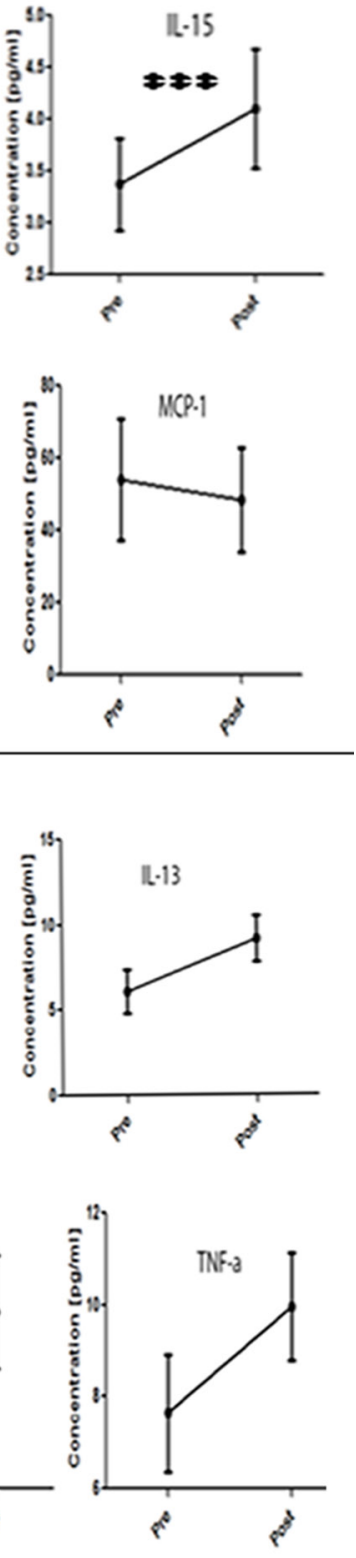

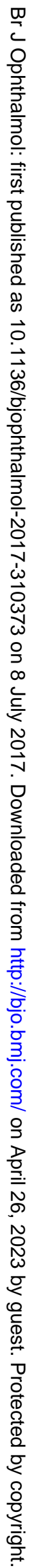

Figure 2 Cytokines expressions premucous membrane grafting (MMG) and post-MMG. (A) Cytokines overexpressed pre-MMG. (B) Cytokines underexpressed pre-MMG. ${ }^{* * *} \mathrm{p}<0.001$. bFF, basic fibroblast growth factor; GM-CSF, granulocyte-macrophage colony-stimulating factor; IL, interleukin; IFN- $\gamma$, interferon- $\gamma$, MCP-1, monocyte chemoattractant protein-1; TNF- $\alpha$, tumour necrosis factor- $\alpha$; VEGF, vascular endothelial growth factor.

from a single patient in the acute stage of SJS showed elevated levels of IL-8, IL-6, TNF- $\alpha$ and CCL2 marking the initiation of the inflammatory process. ${ }^{4}$ There are currently no specific antidotes for the chronic ocular sequelae of the disease. A better understanding of the bioclinical changes in these eyes could highlight the pathophysiology and hence the possibility for targeted treatment options. In our earlier study, LMK was noted to be the most common manifestation in these eyes along with other signs like dryness, symblephara and features suggesfive of timbal stem cell deficiency. ${ }^{2}$ The rationale of MMG in eyes with LMK is to eliminate the lid-wiper induced epitheliopthy and continued timbal stem cell damage. Hence, this study focused on the tear and conjunctival cytokine profile in chronic SJS cases with LMK and the alteration in the same following MMG to understand any additional reparative factors contributed by the mucosa. In the chronic stage of SJS, we observed an

Gurumurthy S, et al. Br J Ophthalmol 2018;102:169-176. doi:10.1136/bjophthalmol-2017-310373

173 


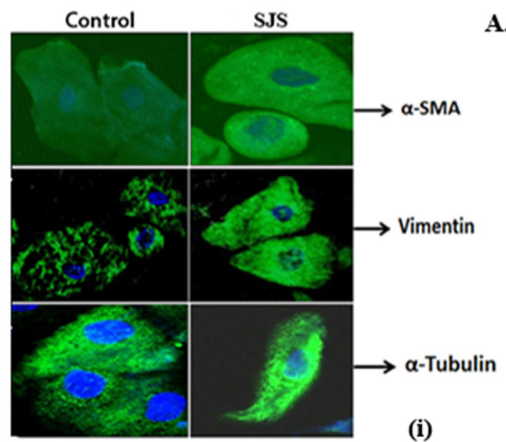

A. EVALUATION OF FIBROTIC MARKERS IN SJS

(i)

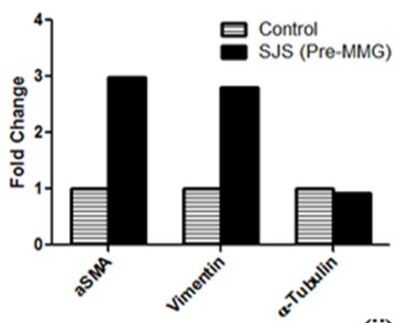

(ii)

B. EVALUATION OF FIBROTIC MARKERS IN SJS PRE AND POST MMG

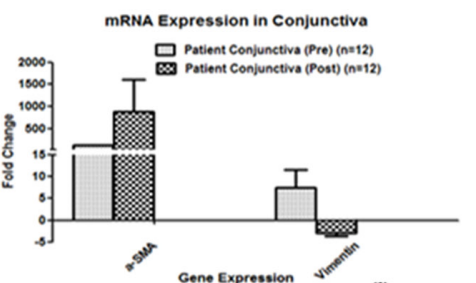

(i)

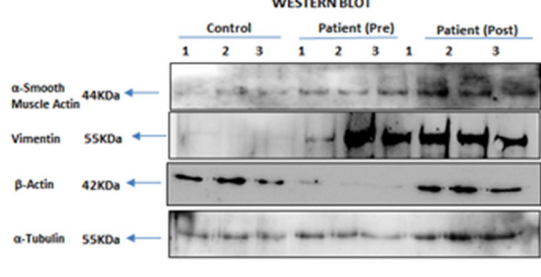

(ii)

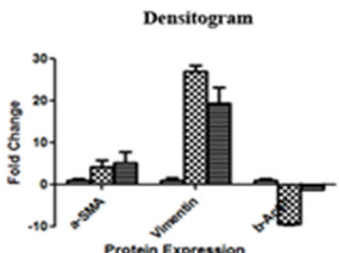

밍 Coetrol Conjunctive Pabent Convinetiva (Post)

(iii)

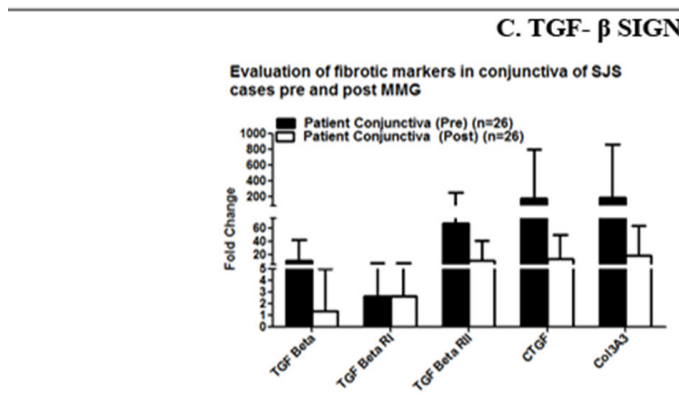

(i)

D. EVALUATION OF APOPTOTIC MARKERS IN SJS PRE AND POST MMG mRNA Expression in Conjunctiva

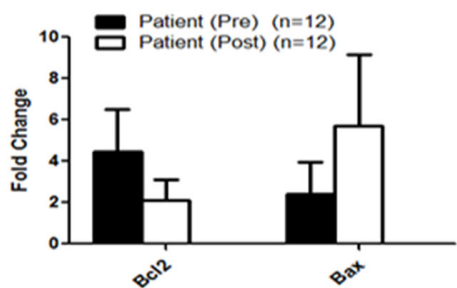

Figure 3 Fibrotic and apoptotic marker expressions in Stevens-Johnson syndrome (SJS) conjunctiva.

altered cytokine profile characterised by dysregulated immune response, decrease of pro-apoptotic activity and an increased fibrotic state (figure 4).

\section{Pro-inflammatory factors}

All the patients with SJS recruited in this study had LMK at the time of presentation and showed a typical pro-inflammatory profile in their tears with increased expressions of GM-CSF, bFGF, IL-8, IL-1 $\beta$, IL-17A and TGF- $\beta 1$. The tear cytokine profile is suggestive of preponderance of fibrosis causing Th17 cells due to persistent inflammation along with lowered expression of the anti-inflammatory cytokines such as IL-10 and IL-13 possibly resulting in LMK. ${ }^{9}$

\section{Profibrotic factors}

A persistently inflammed state is known to cause epithelial-to-mesenchymal transition (EMT) changes, a process that is integral to organogenesis, wound healing and cancer progression. However, uncontrolled EMT changes cause aberrant wound healing by induction of proteases and matrix protein degradation. ${ }^{10} \alpha$-SMA is a specific marker of myofibroblast differentiation in the fibrotic tissue. ${ }^{11}$ This study shows that the potent inducers of EMT, namely, TGF- $\beta 1$, IL-1 $\beta$, IL-8, IL-17A are upregulated. The chronic SJS cases in our study showed fibrosis as seen by increased expressions of CTGF and collagen-III as well as mesenchymal markers vimentin and $\alpha$-SMA in the imprints of conjunctival cells. $\beta$-Actin expression was found to be lost in the conjunctival cells of the chronic SJS. Poor $\beta$-actin expression reported in myofibroblasts is associated with impaired repair mechanism. ${ }^{12}$

\section{Antifibrotic/anti-apoptotic factors}

Furthermore, the antifibrotic cytokines IP-10, IFN- $\gamma$, IL-12p70 and TNF- $\alpha$ that are known to limit fibrosis as reported in 


\section{Cytokine Profile in SJS}

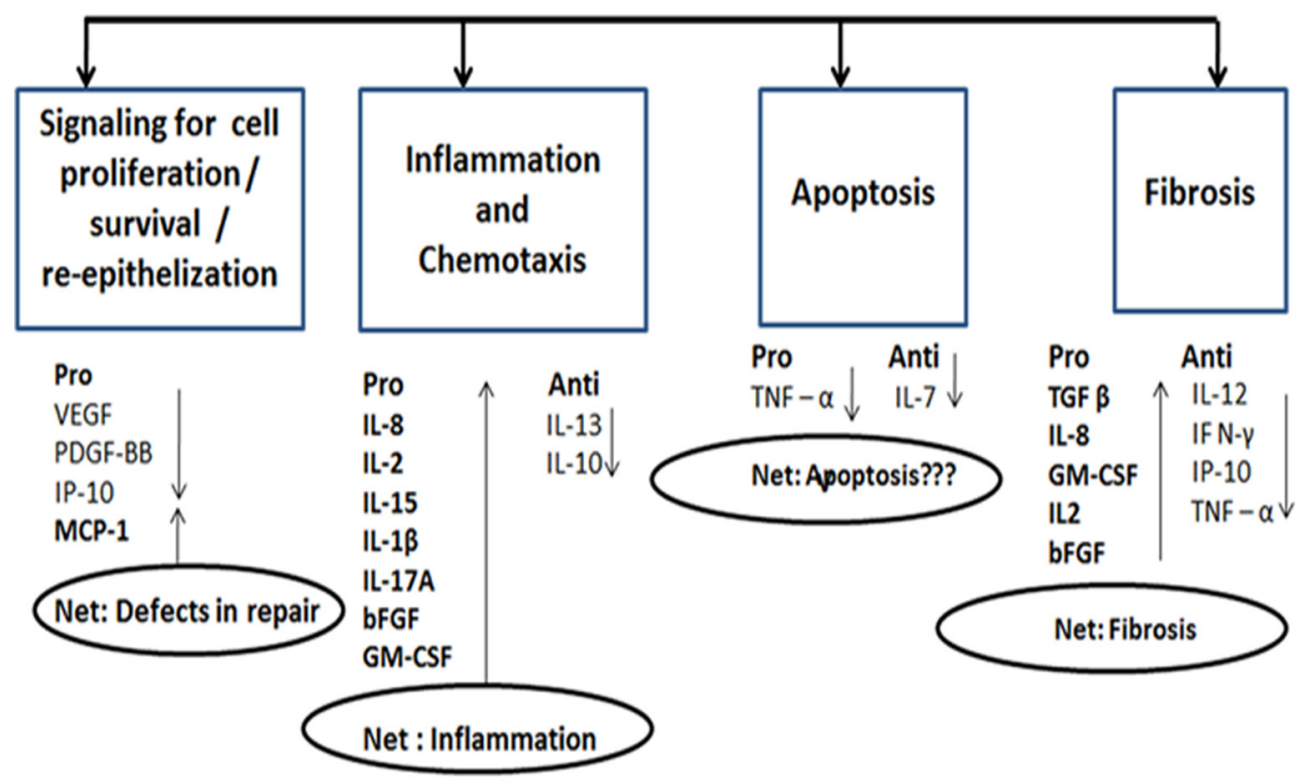

\section{Dysregulated immune response in SJS involves \\ - Inflammation \\ - Defect in re-epithelialization \\ - Apoptosis \\ - Fibrosis}

Figure 4 Summary of the ocular cytokine profile in Stevens-Johnson syndrome (SJS) observed in this study. Arrow indicates their upregulated/ downregulated expression. bFGF, basic fibroblast growth factor; GM-CSF, granulocyte-macrophage colony-stimulating factor; IL, interleukin; IFN- $\gamma$, interferon- $\gamma$, MCP-1, monocyte chemoattractant protein-1; TGF- $\beta$, transforming growth factor-beta; TNF- $\alpha$, tumour necrosis factor-alpha; VEGF, vascular endothelial growth factor.

bleomycin-induced pulmonary fibrosis, ${ }^{13-15}$ were downregulated, clearly favouring the fibrotic events characteristic of chronic SJS. These specific changes in the tear cytokine are contrary to the findings in chronic dry eye disorders, where these factors tend to be upregulated. Although the cutaneous expression of TNF- $\alpha$ is reported to be high in SJS/TEN, ${ }^{16}$ the tear TNF- $\alpha$ levels were found to be lowered in the SJS cases in this study. Macrophage in prohealing phenotype has been associated with lowering of TNF- $\alpha$ and this can induce a profibrotic process. ${ }^{17} 18$ The decreased TNF- $\alpha$ and increased Bcl2 expression seen in this study are indicative of resistance to apoptosis of the myofibroblasts. Anti-TNF- $\alpha$ therapy for SJS management has met with limited success suggesting a possible role of TNF- $\alpha$ in the resolution of fibrosis in the chronic stage. ${ }^{19}$ Conversely, TNF- $\alpha$ antagonists have been shown to be successful in treating fibrotic conditions such as rheumatoid arthritis and systemic sclerosis. $^{20}$

A recently published report on IP-10 in SJS (CXCL10) has shown significantly elevated serum levels in the acute phase of SJS along with elevated IFN- $\gamma^{21}$ Elevated levels are known to occur in tears in chronic inflammatory dry eye disease. ${ }^{22}$ Our study for the first time reports significantly lowered tear IP-10 levels in the chronic state of SJS. This is similar to the reported finding of lowered IP-10 in tears in chronic graft-versus-host disease (cGVHD), wherein the chronic stage of the disease is associated with severe dry eye and subconjunctival fibrotic changes akin to seen in chronic stage of SJS. ${ }^{23}$ The decreased
TNF- $\alpha$ along with reduced IFN- $\gamma$ and IP-10 in extreme cases, could lead to a severe smouldering inflammation and progressive subconjunctival fibrosis mimicking the clinical features of ocular cicatricial pemphigoid.

\section{Post-MMG alteration in cytokine profile}

Post-MMG, the levels of most of the pro-inflammatory cytokines declined with a concomitant improvement in the anti-inflammatory ones. Sustained increase of $\alpha$-SMA post-MMG indicates the proliferative phase of wound healing. This probably is downregulated with time that needs evaluation in further follow-ups. IFN- $\gamma$ which is lowered in SJS pre-MMG improves fivefold with MMG intervention. IFN- $\gamma$ is reported to exert anti-inflammatory effects by various mechanisms including the suppression of differentiating monocyte/macrophages, ${ }^{24}$ inhibits IL-1 $\beta$-mediated production of matrix metalloproteinases and suppress IL-17. ${ }^{25}$ Additional studies are warranted on the role of TNF- $\alpha$ and IFN- $\gamma$ in the acute versus chronic stages of SJS for therapeutic implications.

\section{Role of oral mucosal grafting}

The macrophage functional phenotype can be altered with alteration in the microenvironment. With appropriate stimulus, it can result in altered production of cytokines, metabolites and enzymes that can have a healing outcome. ${ }^{26}$ Oral mucosal squamous epithelial cells are known to have a unique 
immune homeostasis in the oral cavity, where severe inflammatory responses in the oral cavity are relatively rare and tolerance to microbial load is relatively high. ${ }^{27}$ The exact mechanism by which MMG impacts the ocular surface besides eliminating the mechanical lid-wiper effect of LMK is yet to be studied in detail, although our study has shown the profibrotic factors to be much lower in the oral mucosa of patients with SJS compared with the conjunctiva.

\section{Salient findings of this study}

The principal findings of this study are: (1) profibrotic, pro-inflammatory, anti-apoptotic baseline ocular surface milieu with significantly decreased IP-10, TNF- $\alpha$ and IFN- $\gamma$ levels, which could therefore be a potential candidate/s in chronic SJS for targeted therapy; (2) reversal of most of the cytokine profile following MMG, correlating with the clinical improvement; (3) contribution of antifibrotic/anti-inflammatory factors by the oral mucosal niche; (4) an increase in goblet cell density following MMG, the exact underlying mechanism of which is yet to elucidated.

Limitations of this study include lack of corroboration with the serum cytokine profile in the chronic stage and a comparison with other cicatricial conditions like ocular cicatricial pemphigoid, although similar findings have been documented in cGVHD,${ }^{21}$ a condition not so commonly seen in our setting, indicative of a possible similar common pathway in the chronic stage of ocular cicatricial conditions, which are preceded by an acute event. However, further studies are required to understand the switch from the acute to chronic stage, in both the tears and serum.

\section{CONCLUSION}

The ocular surface biochemical profile in chronic SJS appears to be distinct from that in the acute stage. With the profile of the oral mucosa noted in our study, grafting of the oral mucosa could create a new niche beneficial to the conjunctiva by altering its milieu so as to contribute, in addition to elimination of the mechanical sandpaper effect of the LMK, an improvement in the inflammation and decrease in fibrosis, features that constitute the hallmark of chronic SJS.

Acknowledgements The authors acknowledge International Centers for Excellence in Research at National Institute for Research in Tuberculosis, Chennai for the permission to use multiplex reader facility.

Contributors SG contributed to the study protocol, conducted the experiment, analysed and interpreted the data, wrote, proofed and revised the article. AN designed the study protocol, analysed and interpreted the data, wrote, proofed and revised the article. GI and BS provided materials, designed the study protocol, analysed and interpreted the data, proofed and revised the article. SA provided materials, analysed the data, proofed and revised the article.

Funding The study is partly funded by the Department of Science \&Technology SR/ WOS(A)/ LS-1221/2014(G), Government of India.

Competing interests None declared.

Patient consent An institutional consent form was signed by all patients included, agreeing to be part of the study.

Ethics approval Ethics Sub-Committee (Institutional Review Board) 388-2013-P.

Provenance and peer review Not commissioned; externally peer reviewed.

(c) Article author(s) (or their employer(s) unless otherwise stated in the text of the article) 2018. All rights reserved. No commercial use is permitted unless otherwise expressly granted.

\section{REFERENCES}

1 Kohanim S, Palioura S, Saeed HN, et al. Acute and chronic ophthalmic involvement in Stevens-Johnson syndrome/toxic epidermal necrolysis - a comprehensive review and guide to therapy. li. ophthalmic disease. Ocul Surf 2016;14:168-88.

2 Iyer G, Srinivasan B, Agarwal S, et al. Comprehensive approach to ocular consequences of Stevens Johnson syndrome - the aftermath of a systemic condition. Graefes Arch Clin Exp Ophthalmol 2014;252:457-67.

3 Iyer G, Pillai VS, Srinivasan B, et al. Mucous membrane grafting for lid margin keratinization in Stevens-Johnson syndrome: results. Cornea 2010;29:146-51.

4 Yagi T, Sotozono C, Tanaka M, et al. Cytokine storm arising on the ocular surface in a patient with Stevens e Johnson syndrome authors' response. Br J Ophthalmol 2011;95:1-3.

5 Kheirkhah A, Blanco G, Casas V, et al. Surgical strategies for fornix reconstruction based on symblepharon severity. Am J Ophthalmol 2008;146:266-75.

6 Saijyothi AV, Angayarkanni N, Syama C, et al. Two dimensional electrophoretic analysis of human tears: collection method in dry eye syndrome. Electrophoresis 2010;31:3420-7.

7 Gadkari SS, Adrianwala SD, Prayag AS, et al. Conjunctival impression cytology--a study of normal conjunctiva. J Postgrad Med 1992;38:21-3. 22A-22B.

8 Caproni M, Torchia D, Schincaglia E, et al. Expression of cytokines and chemokine receptors in the cutaneous lesions of erythema multiforme and Stevens-Johnson syndrome/toxic epidermal necrolysis. Br J Dermatol 2006;155:722-8.

9 Wilson NJ, Boniface K, Chan JR, et al. Development, cytokine profile and function of human interleukin 17-producing helper T cells. Nat Immunol 2007:8:950-7.

10 Schenke-Layland K, Xie J, Magnusson M, et al. Lymphocytic infiltration leads to degradation of lacrimal gland extracellular matrix structures in NOD mice exhibiting a Sjögren's syndrome-like exocrinopathy. Exp Eye Res 2010;90:223-37.

11 Vozenin MC, Lefaix JL, Ridi R, et al. The myofibroblast markers $\alpha$-SM actin and $\beta$-actin are differentially expressed in 2 and 3-D culture models of fibrotic and normal skin. Cytotechnology 1998;26:29-38.

12 Bunnell TM, Burbach BJ, Shimizu Y, et al. $\beta$-Actin specifically controls cell growth, migration, and the G-actin pool. Mol Biol Cell 2011;22:4047-58.

13 Keane MP, Belperio JA, Burdick MD, et al. IL-12 attenuates bleomycin-induced pulmonary fibrosis. Am J Physiol Lung Cell Mol Physiol 2001;281:L92-7.

14 Pociask DA, Chen K, Choi SM, et al. $\delta$ T cells attenuate bleomycin-induced fibrosis through the production of CXCL10. Am J Pathol 2011;178:1167-76.

15 Redente EF, Keith RC, Janssen W, et al. Tumor necrosis factor- a accelerates the resolution of established pulmonary fibrosis in mice by targeting Pro fi brotic lung macrophages. 2014;50:825-37.

16 Caproni M, Torchia D, Schincaglia E, et al. Expression of cytokines and chemokine receptors in the cutaneous lesions of erythema multiforme and Stevens-Johnson syndrome/toxic epidermal necrolysis. Br J Dermato/ 2006;155:722-8.

17 Greenhalgh DG. The role of apoptosis in wound healing. Int J Biochem Cell Biol 1998;30:1019-30.

18 Johnson A, DiPietro LA. Apoptosis and angiogenesis: an evolving mechanism for fibrosis. Faseb J 2013;27:3893-901.

19 Namazi MR. Increased mortality in toxic epidermal necrolysis with thalidomide: corroborating or exonerating the pathogenetic role of TNF-alpha? Br J Dermatol 2006;155:842-3

20 Distler JH, Schett G, Gay S, et al. The controversial role of tumor necrosis factor alpha in fibrotic diseases. Arthritis Rheum 2008;58:2228-35.

21 Wang F, Cai R, He D, et al. Serum IFN- $\gamma$-inducible chemokines CXCL9 and CXCL10 are elevated in non-immediate drug hypersensitivity reactions. Asian Pac J Allergy Immunol 2016;34:236-41.

22 Liu M, Guo S, Hibbert JM, et al. CXCL10/IP-10 in infectious diseases pathogenesis and potential therapeutic implications. Cytokine Growth Factor Rev 2011;22:121-30.

23 Cocho L, Fernández I, Calonge $\mathrm{M}$, et al. Biomarkers in ocular chronic graft versus host disease: tear cytokine- and chemokine-based predictive model. Invest Ophthalmo/ Vis Sci 2016;57:746

24 Arango Duque G, Descoteaux A. Macrophage cytokines: involvement in immunity and infectious diseases. Front Immunol 2014;5:491.

25 Page CE, Smale S, Carty SM, et al. Interferon-gamma inhibits interleukin-1 betainduced matrix metalloproteinase production by synovial fibroblasts and protects articular cartilage in early arthritis. Arthritis Res Ther 2010;12:R49.

26 Stout RD, Jiang C, Matta B, et al. Macrophages sequentially change their functional phenotype in response to changes in microenvironmental influences. J Immunol 2005; 175:342-9.

27 Novak N, Haberstok J, Bieber T, et al. The immune privilege of the oral mucosa. Trends Mol Med 2008;14:191-8. 